\title{
Cuestionarios sobre factores de riesgo de la exposición y la accidentalidad por tráfico en conductores. Una revisión
}

\section{A review of questionnaires exploring driver's exposure and risk factors in road crashes}

\author{
E. Jiménez Mejías ${ }^{1,2}$, P. Lardelli1,2, C. Amezcua ${ }^{1}$, J.J. Jiménez Moleónn ${ }^{1,2}$
}

\section{RESUMEN}

Dada la diversidad de cuestionarios empleados en el estudio de la epidemiología analítica de las lesiones por tráfico, realizamos una revisión de los estudios publicados al respecto entre 1989 a 2011, con objeto de identificar sus fortalezas y debilidades. Dicha revisión puso de relieve que la mayor parte de los cuestionarios se centran en el factor humano sobre el riesgo de accidente, pero son muy pocos los que incluyen la intensidad de exposición y la asociación de ésta con otros factores de riesgo de la accidentalidad. Muchos cuestionarios poseen un elevado número de ítems y complejas escalas de valoración. Además, en España son escasos los cuestionarios validados sobre la exposición y la accidentalidad por tráfico. Por todo ello, es necesario trabajar en el diseño y la validación de cuestionarios que recojan de forma sencilla información integral sobre la epidemiología de las lesiones por tráfico de cara a su mayor conocimiento y prevención.

Palabras clave. Accidentes de tráfico. Comportamientos de riesgo al volante. Cuestionarios. Escalas. Jóvenes conductores.

\begin{abstract}
Given the diversity of questionnaires currently being used in the study of the analytic epidemiology of traffic injuries, we made a review of studies on this question published between 1989 and 2011 in order to identify their advantages and disadvantages. We were able to observe that most of the questionnaires were focused on the human factor regarding the risk of road crashes, but very few of them included intensity of exposure and its association with other risk factors in road crashes. Many questionnaires have a high number of items and complex rating scales. Furthermore, in Spain there are few validated questionnaires which contain complete information about exposure and road crashes. In Spain we should work on the design and validation of questionnaires containing questions aimed at obtaining complete and easy information about the epidemiology of traffic injuries. In this way we would be able to increase our awareness of traffic injuries and how to prevent them.
\end{abstract}

Key words. Road crashes. Risky driving behaviour. Questionnaires. Scales. Young drivers.
1. Departamento de Medicina Preventiva y Salud Pública. Universidad de Granada.

2. CIBER de Epidemiología y Salud Pública, Spain (CIBERESP).

Recepción: 1 de junio de 2011

Aceptación provisional: 4 de julio de 2011

Aceptación definitiva: 20 de julio de 2011.

\section{Correspondencia:}

Eladio Jiménez Mejías

Departamento de Medicina Preventiva y Salud Pública

Universidad de Granada. Facultad de Medicina Avda. de Madrid, 11

18071 Granada

E-mail: eladiojimenez@ugr.es 


\section{INTRODUCCIÓN}

Es bien conocido que las Lesiones a Consecuencia del Tráfico (LCT) constituyen un problema de salud pública de primera magnitud. La Organización Mundial de la Salud prevé que en el año 2030 sean la $5^{\text {a }}$ causa de mortalidad a nivel mundial y actualmente las sitúa como la primera causa de muerte en población de 15 a 29 años $^{1,2}$. Pese a su magnitud y trascendencia, son varias las organizaciones y autores que las definen como uno de los problemas "más olvidados" en epidemiología ${ }^{3,4}$ a pesar de que el método epidemiológico les es plenamente aplicable. Desde esta perspectiva, las LCT pueden ser modelizadas como una cadena causal constituida por cuatro eslabones secuenciales: exposición, accidentalidad, lesividad y desenlace (mortalidad, incapacidad, etc.).

Para identificar los factores de riesgo que inciden sobre los dos primeros eslabones (la tasa de exposición, usualmente valorada como el número de kilómetros recorridos en un año por los conductores, y el riesgo de sufrir un accidente de tráfico, a igualdad de exposición) es habitual recoger información mediante encuestas, en la que se exploran diversas dimensiones teóricamente asociadas a alguno de los dos componentes anteriores: personalidad, experiencia, percepción del riesgo, distracciones, errores durante la conducción, comisión de infracciones, etc. Ante la gran diversidad de cuestionarios utilizados en la bibliografía, nos ha parecido oportuno hacer una revisión de la misma, cuyo objetivo ha sido identificar las características esenciales -fortalezas y debilidades- de los principales cuestionarios que valoran los potenciales factores de riesgo de la exposición y la accidentalidad en conductores, principalmente de turismos, al objeto de que ello pueda servir de guía para futuras investigaciones sobre este tema.

\section{MATERIAL Y MÉTODOS}

Se realizó una revisión de los estudios publicados sobre LCT en dos fases; en primer lugar, a partir de los trabajos identifi- cados en las bases de datos on-line de Medline (http://www.ncbi.nlm.nih.gov/pubmed), EMBASE (http://www.embase.com), Global Health (OVID-SP) (http://ovidsp. tx.ovid.com/sp-3.4.1b/ovidweb.cgi), Cochrane library (http://www.bibliotecacochrane.com/) y Scopus (http://www.scopus.com/home.url). Además, se realizó una búsqueda en web específicas como SafetyLit Injury Prevention Literature (http://www.safetylit.org/index.htm) y la fundación Real Club del Automóvil de Cataluña (RACC) (http://www.racc.es/index. racc/mod.serviciosHome/mem.lstRevista/ menuA.377/ chk.4404b6fdacc0381af1740c 5a94926900.html?relmenu=1113). En una segunda fase, para aumentar el número de estudios e incluir aquellos no identificados con la estrategia de búsqueda inicial ${ }^{5}$, se seleccionaron trabajos a partir de las referencias bibliográficas de los estudios identificados en la primera fase. Como términos de búsqueda se utilizaron: driving questionnaire; driving scale behaviors and attitudes; road traffic crashes, risky driving styles; driver's behavior pattern; young drivers; $y$ self-report traffic offences o sus equivalentes en castellano: cuestionarios en conductores; escalas sobre comportamientos y actitudes al volante; accidentes de tráfico; estilos de conducción de riesgo; patrones de comportamiento al volante; jóvenes conductores y autodeclaración de infracciones de tráfico.

Los criterios de inclusión empleados para la selección de estudios fueron: 1) Fecha de publicación posterior a 1 de enero de 1985; 2) Trabajos originales; 3) Conductores como población objeto de estudio, en especial, conductores de vehículos de 4 ruedas a motor; 4) Empleo de cuestionarios originales como fuente de información; 5) Información clara y explícita sobre la metodología de recogida de información y 6) Idioma de publicación inglés o español.

Se excluyeron los cuestionarios que exploraran de forma preferente o exclusiva aptitudes físicas del conductor (basados en exploraciones físicas) o dimensiones psíquicas (basados en escalas psicométricas). 
Para cada cuestionario se evaluó la población diana de conductores elegida (total, o definida por variables como la edad, el sexo, o tipo de vehículo), forma de administración del cuestionario (autoadministrado, telefónico o con entrevistador), las dimensiones exploradas, el número de ítems, la forma de valorar las respuestas, la validación original y, en su caso, la validación en población española y el idioma en que están disponibles. Los cuestionarios se han clasificado en dos grandes grupos: los que denominamos integrales (tratan de abarcar todos o la mayoría de factores potencialmente asociados a la exposición y/o accidentalidad), y específicos (centrados en una dimensión concreta de la accidentalidad).

\section{RESULTADOS}

Se identificaron un total de 54 artículos: 43 a través de las diferentes bases de datos y 11 a partir de referencias contenidas en los trabajos inicialmente identificados. El número de trabajos que fueron eliminados fue de 15; 9 por estar duplicados y 6 porque evaluaban en exclusiva aptitudes psicofísicas del conductor. Por tanto, fueron 39 los estudios finalmente incluidos en la revisión.

A continuación se describen los principales cuestionarios empleados en la epidemiología analítica de las LCT, tanto a nivel internacional como nacional. Dedicamos mayor atención a aquellos cuyo empleo ha sido más frecuente por la literatura al respecto.

Estudios internacionales. El primer trabajo que utilizó un cuestionario específico para recoger información sobre actitudes de riesgo relacionadas con la accidentalidad en jóvenes conductores de turismo se realizó a final de la década de los ochenta por Malfetti y col (1989) ${ }^{6}$. Desde el año 1989 hasta abril de 2011 se identificaron un total de 32 cuestionarios o escalas, 29 de ellos clasificados como integrales. En su mayoría son cuestionarios auto-administrados de uso en poblaciones muy diversas, tanto por rango de edad como por tipo de conductor. En un $40 \%$ de los casos no hay información sobre el proceso de validación.

En las tablas 1 y 2 se recogen las características principales de los cuestionarios integrales y específicos de uso más frecuente.

\section{Cuestionarios integrales}

1) El cuestionario más frecuentemente utilizado para conocer las diferentes circunstancias y/o estilos de conducción de riesgo al volante fue el Driver Behaviour Questionnaire (DBQ). La versión original se desarrolló en la Universidad de Manchester en 1990 por Reason y col${ }^{7}$. Sus autores clasificaron empíricamente sus 50 ítems en tres posibles tipos de comportamiento: errores, violaciones y lapsus. Errores, que definieron como fracasos no intencionados en las acciones previstas (por ejemplo no percibir unos peatones que intentan cruzar un paso de cebra). Violaciones, definidas como desviaciones deliberadas de las prácticas de conducción (como conducir por encima de la velocidad permitida) y lapsus, considerados como fallos de memoria y/o de atención con baja probabilidad de afectar a la seguridad durante la conducción (como confundir una dirección $)^{8}$. Posteriormente, Lawton y col diferenciaron dos subtipos de violaciones: las ordinarias (Ordinary violations), relacionadas con las infracciones de las normas de circulación (como conducir muy próximo al vehículo que nos precede), y las interpersonales (Agressive violations) relacionadas con la hostilidad al volante (por ejemplo tocar el claxon o discutir con otros conductores) ${ }^{9}$. 


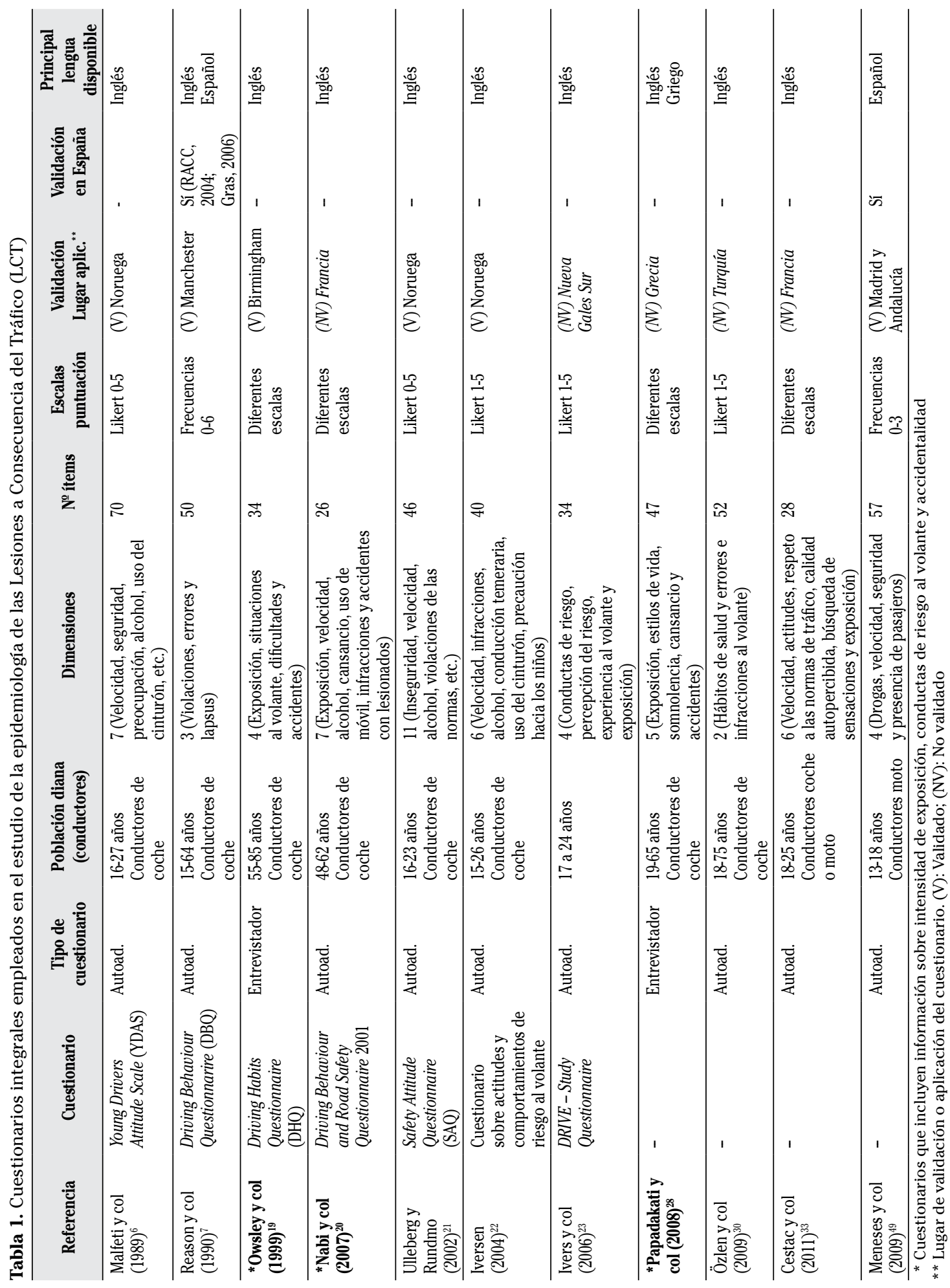




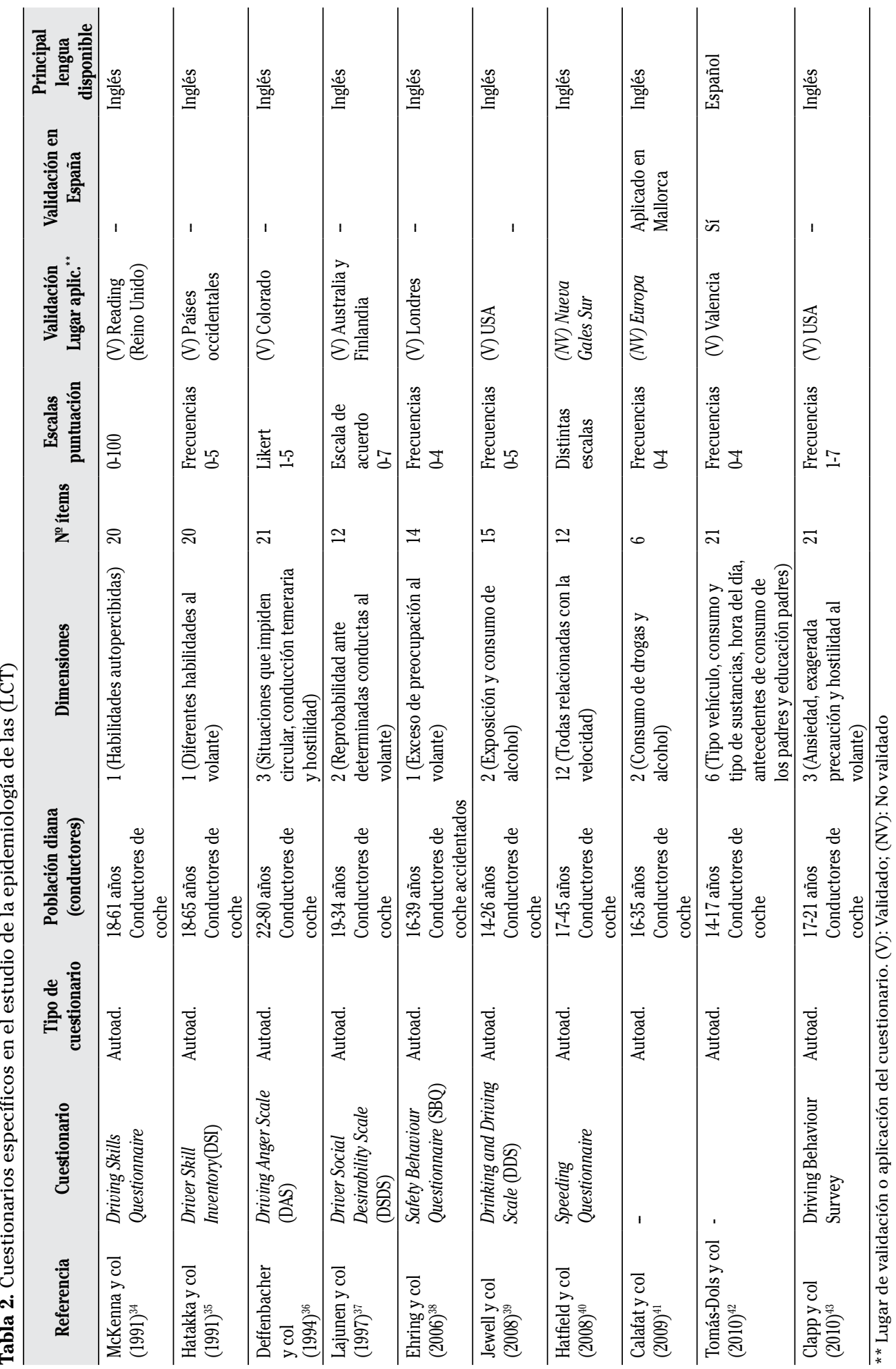


La versión original del DBQ evolucionó bajo dos líneas de trabajo. Por un lado, hacia el desarrollo de versiones más reducidas del cuestionario y su aplicación a un mayor número de conductores ${ }^{10-12}$ y por otro, a la adaptación del mismo a las características sociales y culturales propias de cada país, como su grado de motorización, las características de su red viaria e incluso la diferente percepción de riesgo de ciertas conductas al volante entre países. Así por ejemplo, en Suecia un $71 \%$ de los encuestados contestaron que nunca condujeron tras haber consumido alcohol frente al $8 \%$ obtenido en Italia ${ }^{8}$. Entre los países para los que este cuestionario ha sido adoptado y validado se encuentran Holanda, Finlandia, Suecia, Grecia, China, Turquía, Italia y más recientemente en España ${ }^{7,13-18}$. La versión final consta de 28 ítems agrupados en cuatro dimensiones: violaciones agresivas, infracciones, errores y lapsus.

2) El Young Driver Attitude Scale (YDAS), desarrollado en 1989 por Malfeti y colaboradores para la identificación de actitudes de riesgo en conductores de 16 a 27 años fue, como ya se ha mencionado, el primer cuestionario validado; consta de 70 ítems agrupados en siete dimensiones: 1 . Velocidad, 2. Conducción segura, 3. Montar con un conductor inexperto, 4. Preocupación por otros, 5. Preocupación por uno mismo, 6. Conducir bajo la influencia del alcohol y 7. Uso del cinturón ${ }^{6}$.

3) El Driving Habits Questionnaire (DHQ) de Owsley (1999). Recoge información en conductores adultos y ancianos sobre la intensidad de exposición a la semana (número de lugares visitados, número de kilómetros realizados, etc.), circunstancias durante la conducción (uso del cinturón, velocidad, uso de gafas o lentes de contacto, etc.), dificultades al volante (conducir con lluvia, de noche, solo, etc.) y, finalmente, sobre la accidentalidad (número de accidentes en el último año, número de veces en que fue necesaria la intervención de la policía, etc.) ${ }^{19}$.

4) El Driving Behaviour and Road Safety Questionnaire 2001 (DBRSQ), se empleó en un grupo de 330 conductores de la cohorte multipropósito "GAZEL" en Francia, y ofrece información en el último año sobre: 1. Velocidad, 2. Conducir bajo los efectos del alcohol, 3. Cansancio, 4. Usar el móvil mientras se conduce y 5 . Implicación en 19 infracciones de las normas de circulación ${ }^{20}$.

5) En 2002, Ulleberg y Rundmo desarrollaron un cuestionario sobre actitudes y comportamientos de riesgo durante la conducción. Dicho cuestionario recoge ítems del Safety Attitude Questionnaire (SAQ), que se diseñó para evaluar las actitudes hacia la seguridad y la prevención de accidentes en general. Además, emplearon alguna de las dimensiones e ítems del YDAS. El resultado final fue un cuestionario con 46 ítems distribuidos en 11 dimensiones ${ }^{21}$.

6) Iversen (2004) empleó dos tipos de cuestionarios. Uno, para medir las actitudes, con 16 ítems agrupados en tres dimensiones (1. Actitudes hacia las infracciones de las normas y la velocidad, 2. Actitudes hacia los conductores infractores y 3 . Actitudes hacia el alcohol y la conducción), y otro que medía comportamientos de riesgo al volante. Este último constaba de 24 ítems agrupados en 7 dimensiones (1. Infracciones y velocidad, 2. Conducción temeraria, 3. No uso del cinturón, 4. Vigilancia y precaución al conducir, 5 . Beber y conducir, 6 . Precauciones hacia los niños y 7 . Conducir sobre los límites de velocidad) ${ }^{22}$.

7) El DRIVE Study Questionnaire, desarrollado por Ivers y colaboradores (2006), se aplicó sobre grandes muestras de jóvenes conductores australianos e incluye cuestiones sobre datos sociodemográficos, experiencia al volante (media semanal de horas de conducción), años de antigüedad de permiso, calidad autopercibida como conductor, 14 ítems sobre comportamientos de riesgo y 10 ítems sobre percepción de riesgo ${ }^{23-25}$.

Distintos autores como Laapotti y col (2006), McEvoy y col (2006), Papadakati y col (2008), Chen y col (2009), Özlem y col (2009), Nordfjærn y col (2010), Sündström y col (2010), o Cestac y col (2011), entre otros $^{26-33}$, emplearon cuestionarios integrales, en su mayoría no validados, para explorar diversas dimensiones como comportamientos de riesgo durante la conducción, conducir bajo los efectos del alcohol, habi- 
lidades al volante o haberse visto implicado en uno o más accidentes.

\section{Cuestionarios específicos}

Entre ellos podemos destacar: el Driving Anger Scale (DAS), para evaluar la conducción temeraria y la hostilidad al volante, el Drinking and Driving Scale (DDS) que mide la conducción bajo los efectos de alcohol y otras drogas, o el Speeding Questionnaire que identifica las conductas relacionadas con la velocidad excesiva al volante ${ }^{34-43}$.

Situación en España. En nuestro país, la primera aplicación del DBQ se llevó a cabo en 2004, en un estudio piloto realizado por la Fundación Real Club del Automóvil de Cataluña (RACC) y el Instituto de Tráfico y Seguridad Vial de la Universidad de Valencia (INTRAS). En dicho estudio se empleó una adaptación del cuestionario construida a partir de 126 ítems de las diferentes versiones internacionales ${ }^{44}$.

Gras y colaboradores, en 2006, publicaron un estudio realizado en conductores de entre 22 a 75 años, en el que emplearon una nueva versión adaptada del DBQ. Dicha versión, más simplificada que la del RACC, consta de 27 ítems distribuidos en las 4 dimensiones ${ }^{18}$. También en 2006, López de Cózar y col demostraron la estructura interna del DBQ aplicado a población española ${ }^{45}$. La versión española del DBQ ha sido recientemente empleada en dos estudios llevados a cabo por Gómez-Fraguela y González-Iglesias en 2010. En ambos estudios, el DBQ se empleó conjuntamente con cuestionarios que medían la agresividad y la ira al volante como por ejemplo, el ya mencionado DAS ${ }^{46,47}$.

En el cuestionario bianual utilizado en la cohorte multipropósito SUN (Seguimiento Universidad de Navarra) se han incluido algunas cuestiones relacionadas con la epidemiología de las LCT (número de kilómetros recorridos al año, consumo de alcohol durante la semana, la frecuencia de uso del cinturón y del casco, los accidentes que requirieron hospitalización mayor de 24 horas, etc. $)^{48}$. No obstante, al tratarse de un cuestionario de seguimiento de una cohorte multipropósito, no se recoge información sobre otros muchos factores asociados a la accidentalidad.

Finalmente, Meneses y col (2010), diseñaron un cuestionario con 9 conductas y 7 situaciones de riesgo identificables en adolescentes conductores de moto. Dicho cuestionario fue validado, pudiéndose identificar cuatro dimensiones de agrupación de sus ítems: drogas, velocidad, seguridad y presencia de pasajeros ${ }^{49}$.

\section{DISCUSIÓN}

La mayoría de cuestionarios identificados en esta revisión exploran específicamente factores dependientes del conductor, potencialmente ligados a la accidentalidad, y con frecuencia orientados a poblaciones de conductores jóvenes. Tan sólo los cuestionarios aportados en los trabajos de Owsley y $\mathrm{col}^{19}$, Nabi y $\mathrm{col}^{20}$, Ivers y $\mathrm{col}^{23}$ y Papadakati y $\mathrm{col}^{28}$ y el cuestionario multipropósito de la cohorte SUN, incluyen la intensidad de exposición y la relación de ésta y del factor humano con la incidencia de accidentes, pese a la reconocida importancia de la intensidad de exposición sobre la accidentalidad ${ }^{50}$. A pesar del extenso uso del DBQ para valorar los estilos de conducción de riesgo al volante, algunos estudios ${ }^{8}$ han puesto de relieve sus limitaciones para ser aplicado a países diferentes a los de Europa Occidental, con diferentes tasas de motorización. Este hecho condiciona la mayor o menor exposición a situaciones de riesgo por parte de los conductores y, por ende, la probabilidad de cometer algunos de los errores, lapsus o violaciones que contempla el cuestionario. Otro de los factores que limita la validez externa del DBQ es el diferente grado de reprobación social ante conductas como por ejemplo, conducir bajo los efectos del alcohol entre países. Ya se ha comentado que el $71 \%$ de los conductores suecos manifestaron no haber conducido nunca bajo los efectos del alcohol frente al 8\% de los italianos.

En España es notorio el escaso empleo de cuestionarios de valoración de factores de riesgo de la accidentalidad en conductores. Ello explica la escasa disponibilidad de 
cuestionarios adecuadamente validados. En este sentido, es reseñable la validación del DBQ que realizaron Gras y colaboradores en 2006, si bien, este cuestionario deja al margen un aspecto tan relevante como la intensidad de exposición (kilómetros/año recorridos).

Un aspecto común a la mayoría de cuestionarios revisados es el elevado número de ítems que incorporan y la relativa complejidad de valoración de muchos de ellos, tanto por la formulación de las cuestiones como por la escala de valoración de las respuestas. Indudablemente, todo ello limita la utilidad de estos instrumentos para su uso en grandes muestras de conductores, particularmente en estudios de seguimiento, a la vez que favorece una elevada tasa de no respuesta, potencialmente redundante en sesgos de selección ${ }^{51}$.

Entre las limitaciones de esta revisión se encuentran las derivadas del posible sesgo de selección introducido por los criterios de inclusión definidos, en especial la inclusión de estudios en inglés y español, además del sesgo de publicación inherente a toda revisión.

A partir de todo lo anterior, creemos que nuestro estudio pone de relieve la necesidad en nuestro país de diseñar y validar, o adaptar de entre los ya existentes, un cuestionario que, en la medida de lo posible, reuniera las siguientes características, de cara a su posible empleo en estudios de seguimiento sobre muestras representativas de conductores de todas las edades: auto-cumplimentado, integral (que explore todas las dimensiones ligadas a exposición y accidentalidad) y rápido y sencillo de cumplimentar. Tarea en la que el equipo de investigación epidemiológica de las LCT de nuestro departamento se encuentra trabajando en el momento actual.

\section{BIBLIOGRAFÍA}

1. Organización Mundial de la Salud (OMS). Informe sobre la situación mundial de la seguridad vial: es hora de pasar a la acción. Ginebra: Organización Mundial de la Salud; 2009. Disponible en: www.who.int/violence injury_prevention/road_safety_status/2009.
2. Stevenson MR. Steering in the right direction? Young drivers and road trauma. Med J Aust 2005; 182: 102-103.

3. World Report on Road Traffic Injury Prevention. Geneva: World Health Organization 2004.

4. Seguí-Gomez M, Miller M. Injury prevention and control: reflections on the state and the direction of the field. Salud Pública Mex 2008; 50: 101-111.

5. URRUTIA G, BonfiLl X. Declaración PRISMA: una propuesta para mejorar la publicación de revisiones sistemáticas y metaanálisis. Med Clin (Barc) 2010; 135: 507-511.

6. Malfetti JL, Rose PR, Dekorp NA, Bash CE. The young driver attitude scale. The development and field testing of an instrument to measure young drivers' risk-taking attitudes. New York: New York Teacher Colleague Columbia University 1989.

7. Reason J, Manstead A, Stradling S, Baxter J, CAMPBELL K. Error and violations on the road: A real distinctions? Ergonomics 1990; 33: 1315-1332.

8. Lajunen T, Parker D, Summala H. The Manchester Driver Behaviour Questionnaire: a crosscultural study. Accid Anal Prev 2004; 36: 231238.

9. Lawton T, Parker D, Manstead ASR, Stradling $\mathrm{S}$. The role of affect in predicting social behaviors: the case of road traffic violations. J Appl Soc 1997; 27: 1258-1276.

10. Parker D, McDonald L, Rabbitt P, Sutciffe P. Elderly drivers and their accidents: The aging driver questionnaire. Accid Anal Prev 2000; 32: 751-759.

11. BloKey PN, HaRTLEy LR. Aberrant driving behaviour: errors and violations. Ergonomics 1995; 9: 1759-1771.

12. Sullman MJM, Meadows ML, Pajo KB. Abberrant driving behaviours amongst New Zealand truck drivers. Transport Res Part DTransport Environ 2002; 5: 217-232.

13. ÄBERG L, RimMÖ PA. Dimensions of aberrant driver behaviour. Ergonomic 1998. 41: 39-56.

14. Kontogiannis T, Kossiavelou Z, Marmaras N. Self report of aberrant behaviour on the roads: errors and violations in a sample of Greek drivers. Accid Anal Prev 2002; 34: 381-399.

15. XIE C, PARKER D. A social psychological approach to driving violations in two Chinese cities. Transport Res Part D-Transport Environ 2002; 5: 293-308. 
16. SÜMER, N. Personality and behavioral predictors of traffic accidents: testing a contextual mediated model. Accid Anal Prev 2003; 35 : 949-964.

17. Lucidi F, Giannini AM, Sgalla R, Mallia L, Devoto A, Reichmann S. Young novice driver subtypes: relationship to driving violations, errors and lapses. Accid Anal Prev 2010; 42: 16891696.

18. Gras ME, Sullman MJM, Cunill M, Planes M, Aymerich M, Font-Mayolas S. Spanish drivers and their aberrant driving behaviours. Transport Res Part D-Transport Environ 2006; 9: 129-137.

19. Owsley C, Stalvey B, Wells J, Sloane ME. Older drivers and cataract: driving habits and crash risk. J Gerontol A Biol Sci Med Sci 1999; 54: 203-211.

20. Nabi H, Salmi LR, Lafont S, Chiron M, Zins M, LAGARDE E. Attitudes associated with behavioral predictors of serious road traffic crashes: result from the GAZEL cohort. Inj Prev 2007; 13: 26-31.

21. Ulleberg P, Rundmo T. Risk-taking attitudes among young drivers: The psychometric qualities and dimensionality of an instrument to measure young drivers' risk-taking attitudes. Scand J Psychol 2002; 43: 227-237.

22. IvERSEN H. Risk-taking attitudes and risky driving behaviour. Transport Res Part D-Transport Environ 2004; 7: 135-150.

23. Ivers RQ, Blows SJ, Stevenson MR, Norton RN, Williamson A, Eisenbruch M et al. A cohort study of 20,822 young drivers: the DRIVE study methods and population. Inj Prev 2006; 12: 385-389.

24. Ivers R, Senserrick T, Boufous S, Stevenson M, Chen HY, Woodeward M. Novice drivers' risky behaviour, risk perception, and crash risk: finding from the DRIVE Study. Am J Public Health 2009; 99: 1638-1644.

25. Boufous S, Ivers R, Senserrick T, Stevenson M, Norton R, Williamson A. Accuracy of self-report of on road crashes and traffic offences in a cohort of young drivers: the DRIVE study. Inj Prev 2010; 16: 275-277.

26. LaApotti S, Keskinen E, HatakKa K, HernetKoski K, Katila A, PerÄAho M et al. Driving circumstances and accidents among novice drivers. Traffic Inj Prev 2006; 7: 232-237.

27. McEvoy SP, Stevenson MR, Woodward M. The impact of driver distraction on road safety: results from a representative survey in two Australian states. Inj Prev 2006; 12: 242-247.
28. Chen HY, Ivers RQ, Martiniuk AlC, Boufous S, SEnserrick T, Woodward M et al. Risk and type of crash among young drivers by rurality of residence: Findings from the DRIVE Study. Accid Anal Prev 2009; 41: 676-682.

29. ÖZLEm S, LaJunEn T. Relatinship of seat belt use to health and drivers behaviors. Transport Res Pt F - Traffic Psychol Behav 2009; 12: 235-241.

30. NordFJÆRn T, Jørgensen SH, Rundmo T. An investigation of driver attitudes and behaviour in rural and urban areas. Saf Sci 2010; 48: 348356.

31. Sundström A. The validity of self-reported driver competence: Relations between measures of perceived driver competence and actual driving skill. Transport Res Pt F - Traffic Psychol Behav 2011; 14: 155-163.

32. Cestac J, Paran F, Delhomme P. Young drivers' sensation seeking, subjective norms, and perceived and perceived behavioral control and their roles in predicting intention: How risk-taking motivations evolve gender and driving experience. Saf Sci 2011; 49: 424-432.

33. McKenna, FP. Stanier RA, Lewis C. Factor underlying illusory self-assessment of driving skill in males and females. Accid Anal Prev 1991; 23: 45-52.

34. Hatakka M, Keskinen E, Katila A, LaApotti S. New drivers versus light traffic. Internactional conference on traffic safety; New Delhi, India. 1991; pp. 221-224.

35. Deffenbacher J, Oetting E, Lynch R. Development of a driving anger scale. Psychol Rep 1994; 74: 83-91.

36. Lajunen T, Corry A, Summala H, Hartley L. Impression management and self-deception in traffic behaviour inventories. Per Individ Differ 1997; 22: 341-353.

37. Ehring T, Ehlers A, Glucksman E. Contribution of cognitive factors to the prediction of post-traumatic stress disorder, phobia, and depression after road traffic accident. Behav Res Ther 2006; 44: 1169-1716.

38. Jewell JD, Hupp SDA, SEgrist DJ. Assessing DUI risk: examination of the behavious and attitudes driving and drinking scale (BADDS). Addict Behav 2008; 33: 853-865.

39. Hatfield J, Fernandes R, Faunce G, Soames Job RF. An implicit non-self-report measure of attitudes to speeding: Development and validation. Accid Anal Prev 2008; 40: 616-627.

40. Calafat A, Blay N, Juan M, Adrover D, Bellis MA, Hughes $\mathrm{K}$ et al. Traffic risk behaviors at nightlife: driking, taking drugs, driving, and 
use of public transport by young people. Traffic Inj Prev 2009; 10: 16-69.

41. Tomas Dols S, Alvarez González FJ, Llorens Aleixandre N, Vidal-Infer A, Torrijo Rodrigo MJ, VALDERRAMA-ZuRIÁN JC. Predictors of driving after alcohol and drug use among adolescents in Valencia (Spain). Accid Anal Prev 2010; 42: 2024-2029.

42. Clapp JD, Olsen SA, Beck JG, Palyo SA, Grant DM, GudmundsDotTIR B et al. The driving behavior survey: Sacele constructions and validation. J Anxiety Disord 2010; 25: 96-105.

43. Real Club de Automóviles de Cataluña (RACC). Driving behaviour Questionnaire (DBQ). Cuestionario sobre el comportamiento de los conductores. Aplicación, Adaptación y análisis de la versión española del DBQ. 2004. RACC. Disponible en: http://217.75.226.101/ pub /ficheros/actualidad/actualidad_dp_ dbqcastellano_jzq_4c0fda87.pdf

44. López de Cózar E, Molina JG, Chisvert M, SANMARTIN J. Traducción y adaptación del Driver Behaviour Questionnaire a la población española. IV Congreso de Metodología de Encuestas; 2006 20-22 Septiembre; Pamplona 2006.

45. Gómez-Fraguela JA, GonzÁlez-Iglesias B. El papel de la personalidad y la ira en la explicación de las conductas de riesgo al volante en mujeres jóvenes. Anales de Psicología. 2010; 2 : 318-324.

46. González-Iglesias B, Gómez-Fraguela JA. Conductores infractores, ¿Un perfil de conducta desviada?: Análisis de las diferencias y similitudes con una muestra de conductores de población general. Revista española de investigación criminológica [Internet]. 2010 [Citado 2 Jul 2011]; 8 (6): [aprox. 19 p.]. Disponible en: http://www.criminologia.net/ pdf/reic/ano8-2010/a82010art6.pdf

47. Seguí-Gómez M, Palma S, Guillen-Grima F, de IraLA J, Martinez-GonZalez MA. Self-reported drinking and driving amongst educated adults in Spain: The "Seguimiento Universidad de Navarra" (SUN) cohort findings. BMC Public Health 2007; 12: 7-55.

48. Meneses C, Gil E, Romo N. Adolescentes, situaciones de riesgo y seguridad vial. Aten Primaria 2010; 42: 452-458.

49. ELVIK R, VAA T. Factors contributing to road accidents. En: The Handbook of Road Safety Measures. Oxford, 2004, pp. 29-79.

50. Argimón Pallas JM, Jiménez Villa J. Métodos de investigación clínica y epidemiológica. $3^{\underline{a}} \mathrm{ed}$. Madrid: Elsevier 2004. 